\title{
Clinical Symptoms and Characteristics of Hospitalized Patients with Profound Hyponatremia
}

\author{
Nicole Nigro, Bettina Winzeler, Isabelle Suter, Birsen Arici, Martina Bally, \\ Claudine Blum, Philipp Schuetz, Christian Nickel, Roland Bingisser, \\ Andreas Bock, Andreas Huber, Beat Müller, Mirjam Christ-Crain
}

\section{Background \& Aim}

Background:

- Profound hyponatremia, defined as a serum sodium value below $125 \mathrm{mmol} / \mathrm{L}$ is common and occurs in $2-3 \%$ of hospitalized patients.

- Hyponatremia is associated with substantial morbidity and mortality.

- Symptoms of profound hyponatremia vary among patients between non-specific symptoms such as nausea or malaise or acute life threatening brain oedema.

Aim of the study:

- To prospectively assess patients characteristics, symptoms related to hypoantremia, aetiological causes and management in patients with profound hyponatremia admitted in two tertiary care centers in Switzerland

\section{Patients \& Methods}

\section{Setting:}

-Patients admitted to University Hospital of Basel and the Kantonsspital Aarau with a documented profound hyponatremia ( $\mathrm{Na}+<125 \mathrm{mmol} / \mathrm{L})$ were included in the study.

-On admission all vital signs, Glasgow Coma Scale, actual symptoms, complete medical history including comorbidities, current medication and medication before hospitalisation were recorded in a standardised bed-side patients interview.

-Patients were careful monitored during hospitalization and changes in laboratory values and concomitant comorbidities and mortality were recorded. Further a special attention was paid to the initial recorded symptoms and therapy management of hyponatremia.

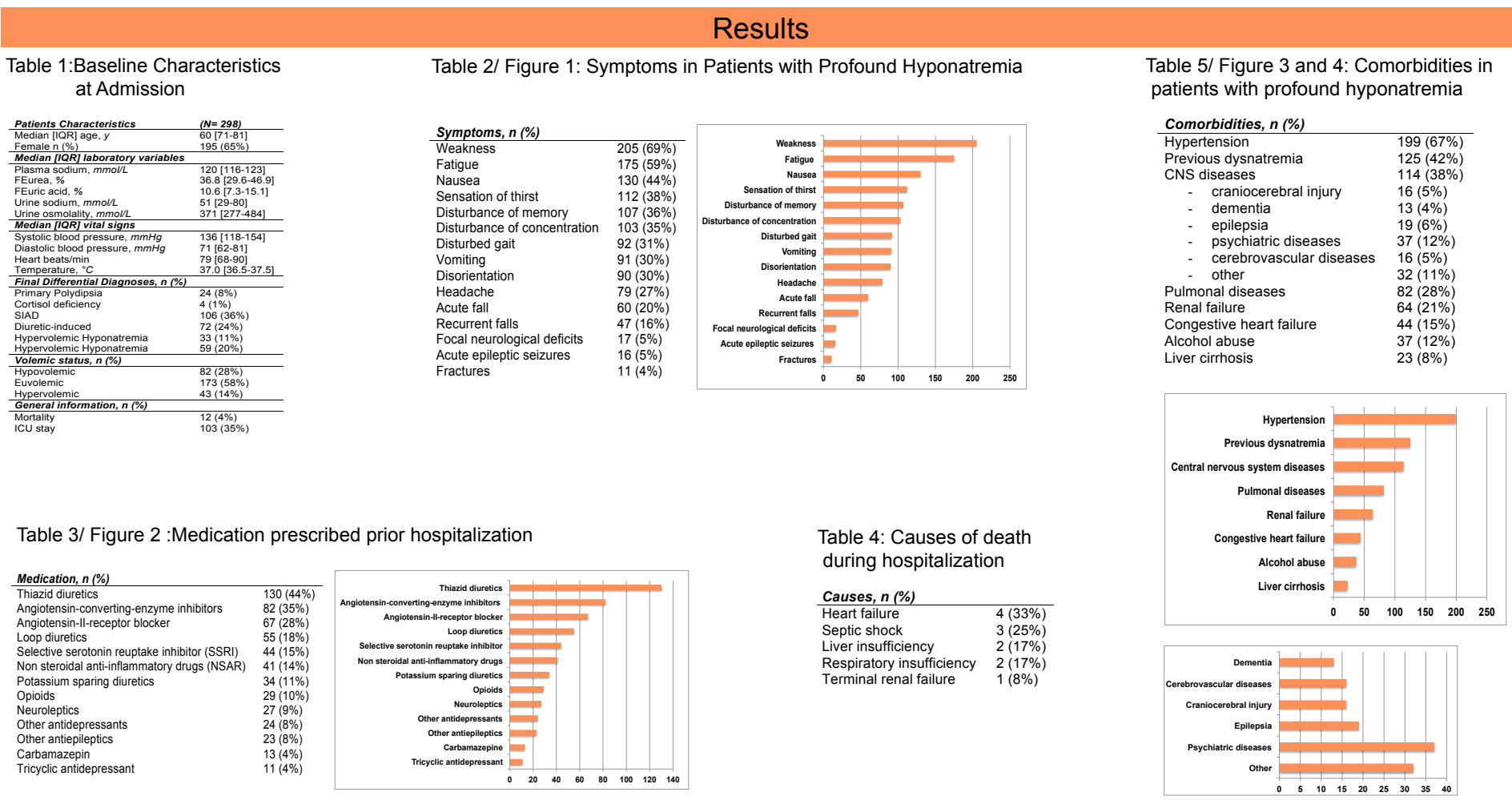

\section{Summary \& Conclusion}

Profound hyponatremia is accompanied by a wide spectrum of different symptoms. Most patients suffered from moderate symptoms reflecting rather chronic hyponatremia with brain cell adaptation. Patients presenting with a profound hyponatremia had several comorbidities and prior medications. 